\title{
Dynamic electrophoresis of droplet dispersions at low surface potentials
}

\author{
Jyh-Ping Hsu, Wei-Lun Min, Eric Lee* \\ Department of Chemical Engineering, Institute of Polymer Science and Engineering, National Taiwan University, Taipei, 10617 Taiwan
}

Received 7 September 2006; accepted 23 October 2006

Available online 28 November 2006

\begin{abstract}
The dynamic electrophoresis of a dispersion of spherical droplets under conditions of low surface potential and arbitrary double-layer thickness and droplet volume fraction is analyzed. A cell model with the Shilov-Zharkikh boundary condition for the electric potential is adopted to simulate a dispersion, and the governing equations and the associated boundary conditions are solved by a pseudo-spectral method based on Chebyshev polynomials. The influence of the frequency of the applied electric field, the volume fraction of the droplets, the thickness of the double layer, and the relative magnitude of the viscosity of the droplet fluid on the electrophoretic behavior of a dispersion is discussed.

(c) 2006 Elsevier Inc. All rights reserved.
\end{abstract}

Keywords: Dynamic electrophoresis; Droplets; Cell model; Low surface potential

\section{Introduction}

The physical properties such as the charge and the linear size of a colloidal particle in a dispersion of appreciable particle concentration can be measured on the basis of the effect of electrokinetic sonic amplitude [1,2]. In this case, because an alternating electric field is applied, both the magnitude and the corresponding phase angle of the electrophoretic mobility of an entity can be measured, achieving more accurate measurement than that when a static electric field is applied. However, compared with the analysis of the latter, that of the former is more complicated, since the time scale is involved. O'Brien [2] investigated the dynamic electrophoresis of a spherical rigid colloid with a very thin double layer in a dilute dispersion. Since this foundation-laying work for theoretical analysis, various studies on dynamic electrophoresis have been carried out in the past two decades [3-17]. Among them, Mangelsdorf and White [4] took into consideration the effect of double-layer polarization. Based on their studies, Ohshima [5] derived an analytic expression for the case of low zeta potential. These studies were all focused on the dilute dispersion, i.e., a spherical particle in an infinite solution. Later, Ohshima extended further to investigate the case of concentrated suspensions. Ac-

\footnotetext{
* Corresponding author. Fax: +886223622530 .

E-mail address: ericlee@ntu.edu.tw (E. Lee).
}

cording to his analysis, the dynamic electrophoretic mobility of the particle could be seriously influenced by the presence of neighboring particles [6-8]. In order to fit real dispersions more closely, various cell models were adopted and results compared [9-11]. Generally speaking, the analytical solution is confined to the limiting case of linearly electrokinetic equations. When a nonlinear effect is involved, for example, the complete nonlinear Poisson-Boltzmann equation, it is difficult to analyze it analytically at arbitrary double-layer thickness and zeta potential. As a result, Lee and co-workers [12-17] applied numerical approach with a pseudo-spectral method to further release this constraint and solved the corresponding general nonlinear equations successfully. They found that the effects of double-layer polarization and overlap are crucial in the dynamic electrophoresis of concentrated dispersions of rigid colloid particles.

The electrophoresis of a dispersion of nonrigid entities has many applications in practice. Emulsions, sols, and foams, for instance, belong to this category. Compared with that of rigid entities, the analysis of the electrophoresis of nonrigid entities is more complicated because the electrokinetic equations for the space inside an entity and those for the space outside an entity need to be solved simultaneously. Several attempts have been made in the literature to model the electrophoretic behavior of a dispersion of nonrigid entities [18-22]. Booth [18] first investigated the electrophoretic behavior of a mercury drop. Later 
on, Levine and O'Brien [20] examined the motion of a droplet or bubble of an emulsion thoroughly and found it can be influenced significantly by an externally applied static electric field. They first introduced the assumption that the polarization effect dominates the electrophoresis of the mercury drop.

While there are many published reports on dynamic electrophoresis, none of them is for nonrigid droplet dispersions; they are essentially all focused on rigid hard particles, to the best of our knowledge. And while there are many published studies on the electrophoresis of droplet dispersions, none of them is focused on dynamic electrophoresis; essentially all of them are confined to static electrophoresis. The only related work in the field of dynamic electrophoresis of nonrigid spheres was published recently by Lee et al. [22], who studied the dynamic electrophoresis of a spherical droplet within a solid cavity. They modeled the electrophoresis in a porous medium with special emphasis on the boundary effect. The polarization effect of a double layer was found to be important.

In this study, we consider dynamic electrophoresis in a concentrated dispersion of spherical droplets when an alternating electric field is applied under conditions of low surface potential and an arbitrary volume fraction of droplets and double-layer thickness. Here, the dispersion is simulated by Kuwabara's unit cell model [23] with the boundary conditions on the cell surface proposed by Shilov and Zharkikh [11]. A pseudo-spectral method based on Chebyshev polynomials used previously for the analysis of electrophoresis under a static applied electric field $[24,25]$ is adopted to solve the resultant governing equations and the associated boundary conditions. The influence of the frequency of the applied electric field, the volume fraction of droplets, the thickness of the double layer, and the relative viscosity of the droplet fluid on the electrophoretic behavior of the dispersion is discussed.

\section{Theory}

Let us consider the electrophoresis of an aqueous dispersion of droplets subject to an alternating electric field. The fluid of a droplet is electrolyte-free, and the dispersion medium contains $z_{1}: z_{2}$ electrolyte, where $z_{1}$ and $z_{2}$ are respectively the valences of cations and anions. We adopt Kuwabara's unit cell model [23], illustrated in Fig. 1, where a dispersion is simulated by a representative droplet of radius $a$ surrounded by a concentric liquid shell of radius $b . a: b$ is chosen to be $1: 2$ in the current calculations. The spherical coordinates $(r, \theta, \varphi)$ are used with origin located at the center of the droplet. $\mathbf{E}=E_{Z} e^{-i \omega t} \mathbf{e}_{\mathbf{z}}$ and $\mathbf{U}=U e^{-i \omega t} \mathbf{e}_{\mathbf{z}}$ are respectively the applied electric field and the corresponding electrophoretic velocity of a droplet, where $\mathbf{e}_{\mathbf{z}}$ is the unit vector in the $Z$-direction. Let $U=U_{\mathrm{R}}+i U_{\mathrm{I}}$, where $U_{\mathrm{R}}$ and $U_{\mathrm{I}}$ are respectively the real and the imaginary parts of $U$ and $i=\sqrt{-1}$.

Since a droplet contains no electrolyte, only the flow field inside it needs to be considered. However, the electric, concentration, and flow fields have to be solved simultaneously for the dispersion medium. In the present problem, the electric field

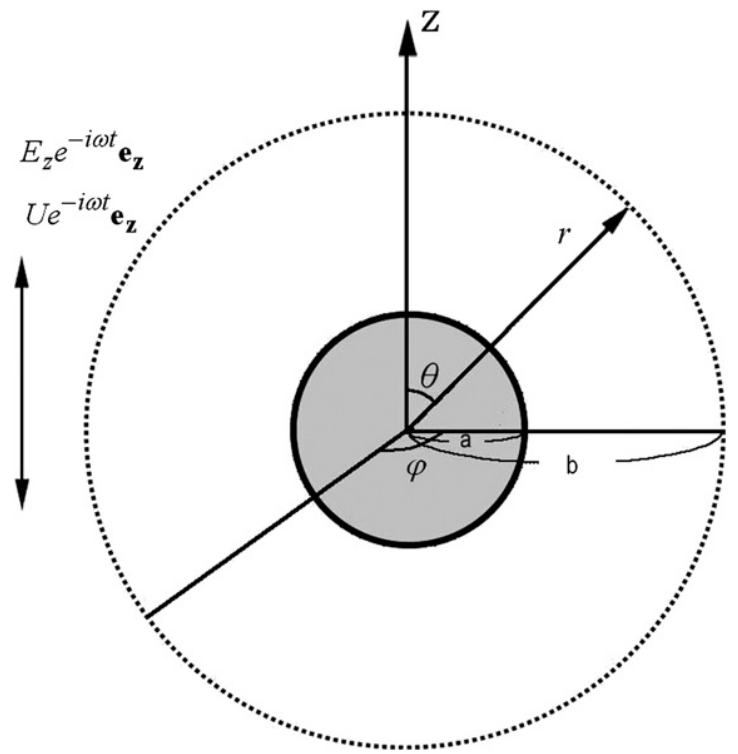

Fig. 1. Kuwabara's unit cell model for a spherical dispersion of droplets of radius $a$, where $b$ is the radius of a concentric liquid shell, $E_{z} e^{-i \omega t} \mathbf{e}_{\mathbf{z}}$ is an applied alternating electric field in the $z$-direction, $U e^{-i \omega t} \mathbf{e}_{\mathbf{z}}$ is the electrophoretic velocity of a droplet, and $(r, \theta, \varphi)$ are the spherical coordinates with origin located at the center of the droplet.

can be derived based on Gauss's law to give

$\nabla^{2} \phi=-\frac{\rho_{\mathrm{e}}}{\varepsilon}=-\sum_{j=1}^{2} \frac{z_{j} \hat{e} n_{j}}{\varepsilon}$,

where $\nabla^{2}$ is the Laplace operator, $\phi$ the electrical potential, $\rho_{\mathrm{e}}$ the space charge density, $\varepsilon$ the dielectric constant of the dispersion medium, $\hat{e}$ the elementary charge, and $n_{j}$ the number concentration of ionic species $j$. The conservation of ionic species $j$ yields

$\frac{\partial n_{j}}{\partial t}=\nabla \cdot\left[D_{j}\left(\nabla n_{j}+\frac{n_{j} \hat{e} z_{j}}{k T} \nabla \phi\right)+n_{j} \mathbf{u}\right]$,

where $\nabla$ is the gradient operator, $D_{j}$ the diffusivity of ionic species $j$, and $\mathbf{u}$ the velocity of the dispersion medium. Since the Reynolds number is very small, the flow field is described by

$\nabla \cdot \mathbf{u}=0, \quad 0<r<b$,

$\rho_{i} \frac{\partial \mathbf{u}}{\partial t}=-\nabla p+\eta_{i} \nabla^{2} \mathbf{u}, \quad 0<r<a$,

$\rho_{0} \frac{\partial \mathbf{u}}{\partial t}=-\nabla p+\eta_{0} \nabla^{2} \mathbf{u}-\rho_{\mathrm{e}} \nabla \phi, \quad a<r<b$.

In these expressions, $p$ is the pressure, $\eta_{i}$ and $\eta_{0}$ are respectively the viscosity of the droplet fluid and that of the dispersion medium, and $\rho_{i}$ and $\rho_{0}$ are respectively the density of the droplet fluid and that of the dispersion medium.

Under conditions of practical significance, the strength of the applied electric field is relatively weaker than that established by the charge on the droplet surface. For convenience, each dependent variable is expressed as the sum of its equilibrium value, that is, the value when the external electric field is absent, and a perturbed value, which arises from the applied 
electric field. Also, because the Reynolds number is very small for the present problem, the droplet remains spherical, and the dependent variables are functions of $r, \theta$, and $t$ only. Therefore,

$$
\begin{aligned}
& \phi(r, \theta, t)=\phi_{\mathrm{e}}(r)+\delta \phi(r, \theta) E_{Z} e^{-i \omega t}, \\
& n_{j}(r, \theta, t)=n_{j}^{\mathrm{e}}(r, \theta)+\delta n_{j}(r, \theta) E_{Z} e^{-i \omega t}, \\
& \mathbf{u}(r, \theta, t)=\mathbf{0}+\delta \mathbf{u}(r, \theta) E_{Z} e^{-i \omega t}, \\
& p(r, \theta, t)=p_{\mathrm{e}}(r, \theta)+\delta p(r, \theta) E_{Z} e^{-i \omega t} .
\end{aligned}
$$

In these expressions, the subscript e and the superscript e denote equilibrium property, and a symbol with $\delta$ represents a perturbed quantity arising from the applied electric field. Note that since a droplet remains stationary when the external electric field is absent, $\mathbf{u}=\mathbf{0}$. Also, $\phi_{\mathrm{e}}$ is a function of $r$ only.

\section{Equilibrium system}

In the absence of the applied electric field, droplets remain stationary, and therefore, only the electric and the concentration fields need to be considered. Since the surface potential is low, the equilibrium potential satisfies the linearized PoissonBoltzmann equation,

$\nabla^{2} \phi_{\mathrm{e}}=\sum_{j=1}^{N} \frac{z_{j} \hat{e} n_{j 0}^{\mathrm{e}}}{\varepsilon}\left(\frac{z_{j} \hat{e} \phi_{\mathrm{e}}}{k T}\right)$,

where $n_{j 0}^{\mathrm{e}}$ is the bulk concentration of ionic species $j$. Suppose that the electric potential remains constant on the droplet surface, and there is no net electric current across the outer virtual cell surface. Then

$\begin{array}{ll}\phi_{\mathrm{e}}=\zeta_{a}, & r=a, \\ \frac{\mathrm{d} \phi_{\mathrm{e}}}{\mathrm{d} r}=0, & r=b .\end{array}$

\section{Perturbed system}

Taking $\nabla^{2}$ on Eq. (6) gives

$\nabla^{2}\left(\delta \phi(r, \theta) E_{Z} e^{-i \omega t}\right)=\nabla^{2} \phi(r, \theta, t)-\nabla^{2} \phi_{\mathrm{e}}(r)$.

Under the condition of low surface potential the perturbed ionic concentration is negligible, $\delta n_{j} E e^{-i w t}=0$, which implies that

$\nabla^{2} \phi(r, \theta, t)=\nabla^{2} \phi_{\mathrm{e}}(r)$.

Therefore

$\nabla^{2}\left(\delta \phi(r, \theta) E e^{-i w t}\right)=0$.

In the absence of $\mathbf{E}$ the fluid is stagnant, $\nabla p_{\mathrm{e}}=0$, and $\mathbf{u}_{\mathbf{e}}=$ $\mathbf{0}$, where $p_{\mathrm{e}}$ and $\mathbf{u}_{\mathrm{e}}$ are respectively the pressure and the fluid velocity at equilibrium. Therefore, only the perturbed flow field needs to be considered. Equations (3)-(6) and (8) yield

$$
\begin{aligned}
\nabla \cdot\left(\delta \mathbf{u} E_{Z} e^{-i \omega t}\right)= & 0, \quad 0<r<b, \\
-i \omega \rho_{0} \delta \mathbf{u} E_{Z} e^{-i \omega t}= & -\nabla \delta p E_{Z} e^{-i \omega t}+\eta_{0} \nabla^{2} \delta \mathbf{u} E_{Z} e^{-i \omega t} \\
& -\rho_{\mathrm{e}} \nabla \phi, \quad a<r<b,
\end{aligned}
$$

$$
\begin{aligned}
& -i \omega \rho_{i} \delta \mathbf{u} E_{Z} e^{-i \omega t}=-\nabla \delta p E_{Z} e^{-i \omega t}+\eta_{i} \nabla^{2} \delta \mathbf{u} E_{Z} e^{-i \omega t}, \\
& 0<r<a .
\end{aligned}
$$

For an easier treatment, the stream function $\psi$ is adopted to describe the flow field. In terms of $\psi$, we have

$$
\begin{aligned}
\delta \mathbf{u} E_{Z} e^{-i \omega t} & =\left(\delta u_{r} \hat{\mathbf{r}}+\delta u_{\theta} \hat{\boldsymbol{\theta}}\right) E_{Z} e^{-i \omega t} \\
& =\left(-\frac{1}{r^{2} \sin \theta} \frac{\partial \psi}{\partial \theta} \hat{\mathbf{r}}+\frac{1}{r \sin \theta} \frac{\partial \psi}{\partial r} \hat{\boldsymbol{\theta}}\right) E_{Z} e^{-i \omega t},
\end{aligned}
$$

where $\delta u_{r}=-\left(1 / r^{2} \sin \theta\right)(\partial \psi / \partial \theta)$ and $\delta u_{\theta}=(1 / r \sin \theta) \times$ $(\partial \psi / \partial r)$ are respectively the $r$ - and the $\theta$-components of $\delta \mathbf{u}$. Note that Eq. (16) is satisfied automatically by these two expressions. Taking the curl on both sides of Eqs. (17) and (18) and introducing the stream function, we obtain

$$
\begin{aligned}
& \eta_{0} E^{4} \psi+i \omega \rho_{0} E^{2} \psi=\left(\frac{\partial \rho_{\mathrm{e}}}{\partial r} \frac{\partial \phi}{\partial \theta}-\frac{\partial \rho_{\mathrm{e}}}{\partial \theta} \frac{\partial \phi}{\partial r}\right) \sin \theta, \\
& a<r<b, \\
& \eta_{i} E^{4} \psi+i \omega \rho_{i} E^{2} \psi=0, z \quad 0<r<a,
\end{aligned}
$$

where $E^{2}=\left(\frac{\partial^{2}}{\partial r^{2}}\right)+\frac{\sin \theta}{r^{2}} \frac{\partial}{\partial \theta}\left(\frac{1}{\sin \theta} \frac{\partial}{\partial \theta}\right)$ and $E^{4}=E^{2} E^{2}$.

For the electric field, because the fluid inside a droplet is electrolyte-free and the surface conductivity is negligible, we assume that the droplet surface is impenetrable to ions; that is,

$\nabla\left(\delta \phi E_{Z} e^{-i \omega t}\right) \cdot \hat{\mathbf{n}}=0, \quad r=a$.

On the cell surface, the boundary condition proposed by Shilov and Zharkikh [11] is adopted; that is,

$\left(\delta \phi E_{Z} e^{-i \omega t}\right)=E_{Z} e^{-i \omega t} b \cos \theta, \quad r=b$.

Since the bulk liquid cannot penetrate a droplet and both the velocity and the shear stress are continuous across the droplet surface, the following conditions are assumed,

$$
\begin{aligned}
& \left.\delta u_{r} E_{Z} e^{-i w t}\right|_{r=a^{+}}=\left.\delta u_{r} E_{Z} e^{-i w t}\right|_{r=a^{-}}=0, \quad r=a, \\
& \left.\delta u_{\theta} E_{Z} e^{-i w t}\right|_{r=a^{+}}=\left.\delta u_{\theta} E_{Z} e^{-i w t}\right|_{r=a^{-}}, \quad r=a, \\
& \left(\underline{\underline{\boldsymbol{\tau}}}^{\mathbf{n}} \cdot \hat{\mathbf{n}}\right) \times\left.\hat{\mathbf{n}}\right|_{r=a^{+}}=\left(\underline{\underline{\boldsymbol{\tau}}}^{\mathbf{n}} \cdot \hat{\mathbf{n}}\right) \times\left.\hat{\mathbf{n}}\right|_{r=a^{-}}, \quad r=a,
\end{aligned}
$$

where $\underline{\underline{\mathbf{n}}}^{\mathbf{n}}$ is the shear stress tensor on the droplet surface. Here, we assume that the cell surface moves with a velocity $-U E_{Z} e^{-i \omega t}$ relative to a droplet. Also, according to Kuwabara [23], the vorticity should vanish on the cell surface. Therefore the following conditions are assumed on the cell surface:

$$
\begin{aligned}
& \nabla \times \delta \mathbf{u} E_{Z} e^{-i \omega t}=0, \quad r=b, \\
& \delta u_{r} E_{Z} e^{-i \omega t}=-\left(U_{\mathrm{R}}+i U_{\mathrm{I}}\right) E_{Z} e^{-i \omega t} \cos \theta, \quad r=b .
\end{aligned}
$$

Furthermore, the symmetric nature of the present problem requires that

$$
\begin{array}{ll}
\delta u_{r} E_{Z} e^{-i w t}=0, & r=0, \\
\delta u_{\theta} E_{Z} e^{-i w t}=0, & r=0 .
\end{array}
$$




\section{Electrophoretic mobility}

For the present case, the electrophoretic mobility $\mu$ is defined by

$\mu=\mu_{\mathrm{R}}+i \mu_{\mathrm{I}}=\frac{U}{E}$,

where $E=E_{Z} e^{-i \omega t}$ and $U=\left(U_{\mathrm{R}}+i U_{\mathrm{I}}\right) E_{Z} e^{-i \omega t}, \mu_{\mathrm{R}}$ and $\mu_{\mathrm{I}}$ being respectively the real and the imaginary parts of $\mu$. To evaluate $\mu$, a force balance on a droplet is conducted to give

$\mathbf{F}_{\mathbf{h}}+\mathbf{F}_{\mathbf{e}}=\frac{4}{3} \pi a^{3}\left(\rho_{i}-\rho_{0}\right) \frac{\mathrm{d} \mathbf{U}}{\mathrm{d} t}$,

where $\mathbf{F}_{\mathbf{h}}$ and $\mathbf{F}_{\mathbf{e}}$ are respectively the hydrodynamic force and the electric force acting on a droplet. For the present problem, only the $z$-components of these forces need to be considered. Since the charge on the droplet surface is distributed uniformly and the droplet fluid is electrolyte-free, the $z$-component of $\mathbf{F}_{\mathbf{e}}$, $F_{\text {e }}$, can be calculated as

$$
\begin{aligned}
F_{\mathrm{e}}= & \iint_{S} \sigma(-\nabla \phi)_{s} \cdot \mathbf{e}_{\mathbf{z}} \mathrm{d} S \\
= & 2 \pi \varepsilon \zeta_{a}^{2} \int_{0}^{\pi}\left(\frac{\partial \phi}{\partial r}\right)_{r=a}\left(\frac{\partial \phi}{\partial r} \cos \theta-\frac{1}{r} \frac{\partial \phi}{\partial \theta} \sin \theta\right)_{r=a} \\
& \times r^{2} \sin \theta \mathrm{d} \theta,
\end{aligned}
$$

where $S$ denotes the droplet surface and $\sigma$ is the surface charge density, which can be determined by Gauss's law. Under a creeping flow condition the $z$-component of $\mathbf{F}_{\mathbf{h}}, F_{\mathrm{h}}$, can be evaluated by [26]

$$
\begin{aligned}
F_{\mathrm{h}}= & -\pi \rho_{0} \int_{0}^{\pi}\left[r^{2} \sin \theta \frac{\partial}{\partial t}\left(\frac{\partial\left(\psi E_{Z} e^{-i \omega t}\right)}{\partial r}\right)\right]_{r=a} \mathrm{~d} \theta \\
& +\eta \pi \int_{0}^{\pi}\left[r^{4} \sin ^{3} \frac{\partial}{\partial r}\left(\frac{E^{2} \psi E_{Z} e^{-i \omega t}}{r^{2} \sin ^{2} \theta}\right)\right]_{r=a} \mathrm{~d} \theta \\
& -\pi \int_{0}^{\pi}\left[r^{2} \sin ^{2} \theta \rho_{\mathrm{e}} \frac{\partial \phi}{\partial \theta}\right]_{r=a} \mathrm{~d} \theta .
\end{aligned}
$$

For an easier treatment, the problem under consideration is decomposed into two subproblems. In the first subproblem a droplet moves with a scaled velocity $\left(U_{\mathrm{R}}^{*}+i U_{\mathrm{I}}^{*}\right) E_{Z}^{*} e^{-i \omega t}$ in the absence of $\mathbf{E}$, and in the second subproblem $\mathbf{E}$ is applied but the droplet remains stationary in space. $E_{Z}^{*}$ represents the scaled electric field. Let $F_{1}^{*}$ and $F_{2}^{*}$ be respectively the scaled total forces in subproblems 1 and 2. Since the influence of the perturbed electric field is of a linear nature, $F_{1}^{*}$ is proportional to $\left(U_{\mathrm{R}}^{*}+i U_{\mathrm{I}}^{*}\right) E_{Z}^{*} e^{-i \omega t}$, and $F_{2}^{*}$ is proportional to $E_{Z}^{*} e^{-i \omega t}$ $E_{Z}^{*} e^{-i \omega t}$; that is,

$F_{1}^{*}=\chi\left(U_{\mathrm{R}}^{*}+i U_{\mathrm{I}}^{*}\right) E_{Z}^{*} e^{-i \omega t}$,

$F_{2}^{*}=\beta E_{Z}^{*} e^{-i \omega t}$ where $\chi$ and $\beta$ are complex constants. In scaled symbols, Eq. (32) can be rewritten as

$$
\begin{aligned}
F_{\mathrm{h}}^{*}+F_{\mathrm{e}}^{*}=F_{1}^{*}+F_{2}^{*}= & -i\left(\frac{4}{3} \pi \varepsilon \zeta_{a}^{2}\right)\left(\frac{\rho_{0} \omega a^{2}}{\eta_{0}}\right)\left(\frac{\rho_{i}-\rho_{0}}{\rho_{0}}\right) \\
& \times\left(U_{\mathrm{R}}^{*}+i U_{\mathrm{I}}^{*}\right) E_{Z}^{*} e^{-i \varpi t} .
\end{aligned}
$$

Based on Eqs. (31) and (35)-(37), a scaled mobility $\mu^{*}=$ $\mu /\left(\varepsilon \zeta_{a} / \eta_{0}\right)$ can be expressed as

$\mu^{*}=\frac{-\beta}{\chi+i\left(\frac{\rho_{0} \omega a^{2}}{\eta_{0}}\right)\left(\frac{\rho_{i}-\rho_{0}}{\rho_{0}}\right)}$.

Here, $\mu^{*}$ is a complex number, which can be characterized by its magnitude $\mu_{m}^{*}=\sqrt{\mu_{\mathrm{R}}^{2}+\mu_{\mathrm{I}}^{2}}$ and phase angle $\Theta=$ $\tan ^{-1}\left(\mu_{\mathrm{I}} / \mu_{\mathrm{R}}\right)$; that is,

$\mu^{*}=\mu_{m}^{*} e^{i \Theta}$.

Since $\mathbf{E}=E_{Z} e^{-i \omega t} \mathbf{e}_{\mathbf{z}}$ and $\mathbf{U}=\sqrt{U_{\mathrm{R}}^{2}+U_{\mathrm{I}}^{2}} E_{Z} e^{-i \omega t+\Theta} \mathbf{e}_{\mathbf{z}}$, $\Theta<0$ implies that $\mathbf{U}$ leads $\mathbf{E}$, and the reverse is true if $\Theta>0$.

The governing equations, Eqs. (10) and (13)-(21), and the associated boundary conditions, Eqs. (11), (12), and (22)-(30), are solved by a pseudo-spectral method based on Chebyshev polynomials, which is found to be efficient and accurate for problems of the present type [24,25].

\section{Results and discussion}

The factors that might influence the electrophoretic behavior of the system under consideration include the scaled frequency $\omega^{*}=\rho_{0} \omega a^{2} / \eta_{0}$, the scaled double-layer thickness $\kappa a$, where $\kappa^{-1}=\left(\varepsilon k T / \sum_{j=1}^{2} n_{j 0}^{e}\left(e z_{j}\right)^{2}\right)^{1 / 2}$, the viscosity ratio $\left(\eta_{0} / \eta_{i}\right)$, the scaled surface potential $\phi_{r}=z_{1} e \zeta_{a} / k T$, and the volume fraction of droplets, which can be measured by $H=(a / b)^{3}$. An aqueous $\mathrm{KCl}$ solution is chosen to illustrate the typical behavior of a dispersion. The following values are assumed in numerical simulations: $T=298.15 \mathrm{~K}, z_{1}=-z_{2}=1 \mathrm{~F} / \mathrm{m}$, $\eta_{0}=8.904 \times 10^{-3} \mathrm{~g} /(\mathrm{cm} \mathrm{s}), \rho_{0} / \rho_{i}=0.909, \zeta_{a}=25.6 \mathrm{mV}$, and $\varepsilon=8.854 \times 10^{-12} \times 78.54688$.

Fig. 2 shows the variations of the scaled magnitude of electrophoretic mobility $\mu_{m}^{*}$ and the corresponding phase angle $\Theta$ as a function of the scaled frequency of the applied electric field $\omega^{*}$ for various values of $H$, and those as a function of $\kappa a$ are presented in Fig. 3. $H$ represents the volume fraction of droplets. For example, if $H=0.1$, the dimensionless distance between droplets, $2(b-a)$, would be approximately 2.3. Double-layer overlapping is allowed in the current analysis. For the configuration under discussion $(a: b=1: 2), \kappa a$ smaller than 1 indicates the occurrence of overlapping, where $1 / \kappa$ is chosen as the index of the double-layer thickness. As can be seen in Fig. 2, for a fixed value of $H$, an increase in $\omega^{*}$ has the effect of reducing $\mu_{m}^{*}$. This is because in Eqs. (20) and (21), as $\omega^{*}$ gets large, the flow field will be dominated by the inertia term, and the influences of the other terms become less important. Moreover, as the direction of the applied electric field changes back and forth, that of the movement of a droplet varies accordingly. Therefore, the higher the $\omega^{*}$ the shorter the time 


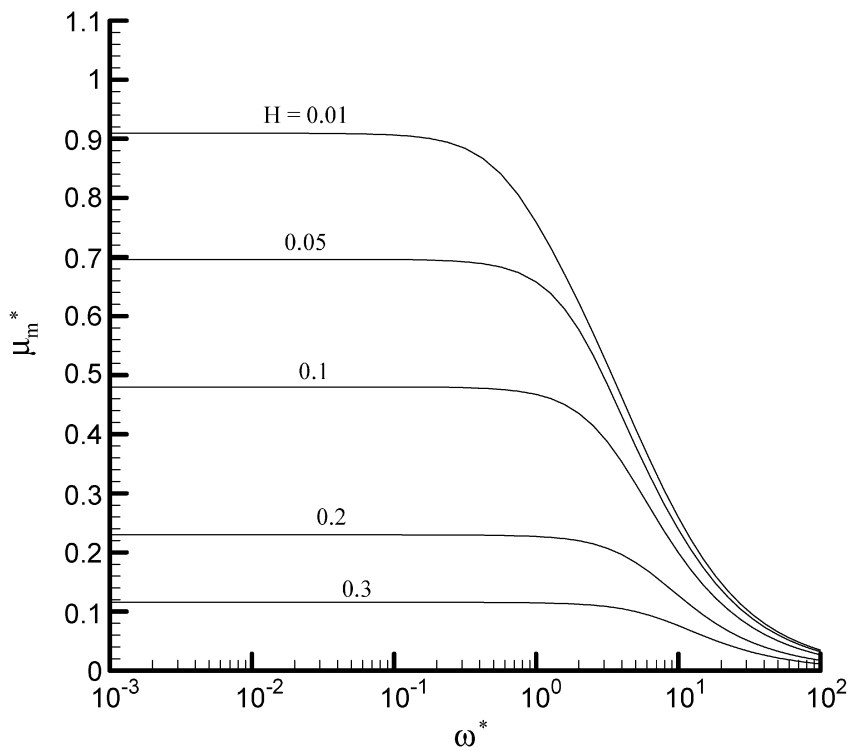

(a)

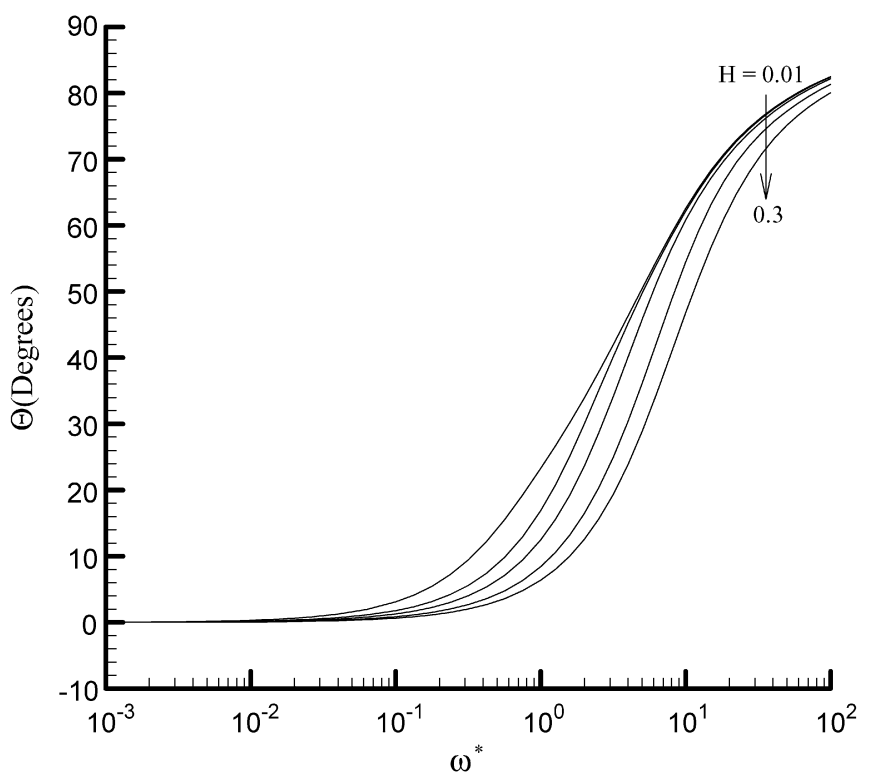

(b)

Fig. 2. Variation of scaled magnitude of electrophoretic mobility $\mu_{m}^{*}$ (a), and phase angle $\Theta(b)$, as a function of scaled frequency $\omega^{*}$ for various values of $H$ at $\kappa a=1$ and $\eta_{0} / \eta_{i}=1$.

available for the acceleration of a droplet, and hence the smaller the mobility. It is expected that as $\omega^{*} \rightarrow \infty, \mu_{m}^{*} \rightarrow 0$; that is, a droplet remains stationary. Fig. 2 also shows that for a fixed level of $\omega^{*}, \mu_{m}^{*}$ decreases with the increase in $H$. This is mainly due to the hindrance effect between neighboring droplets. The higher the volume fraction ratio $H$, the more significant this effect becomes. Fig. 2b reveals that $\Theta>0$ (phase lag) for the present problem. This is because a droplet needs some time to adjust its surrounding ionic cloud so that they can move simultaneously. If $\omega^{*}$ is large, the time for a droplet to react by altering its direction of movement becomes short, and hence the phase lag increases accordingly. The droplets simply run out of time to catch up with the fast-alternating input electric field. The apparent outcome is increasing phase lag, like

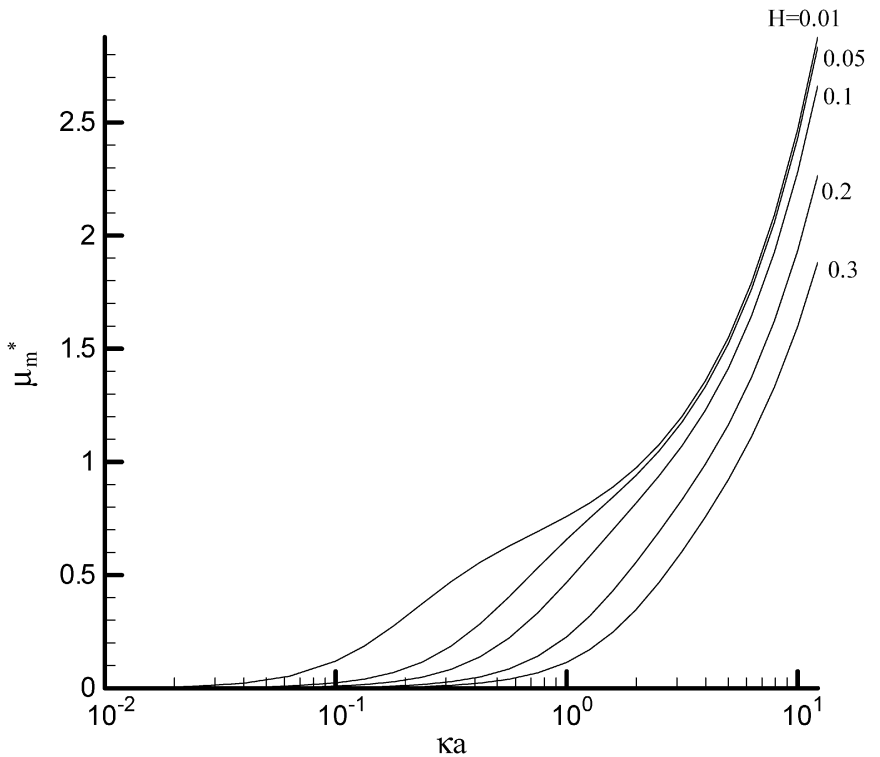

(a)

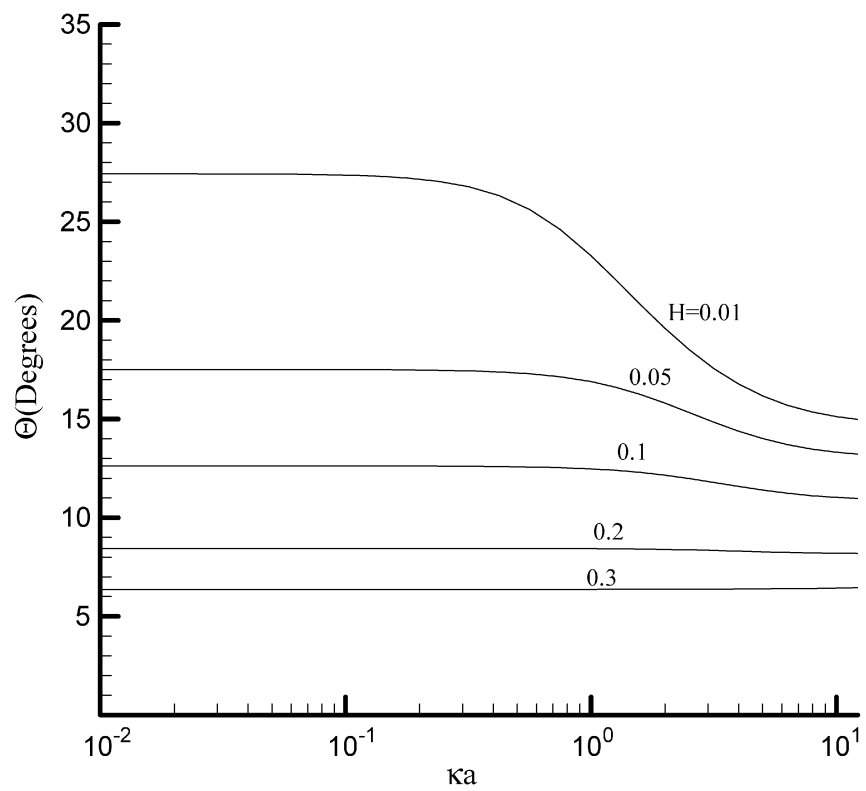

(b)

Fig. 3. Variation of scaled magnitude of electrophoretic mobility $\mu_{m}^{*}$ (a), and phase angle $\Theta(\mathrm{b})$, as a function of $\kappa a$ for various values of $H$ at $\omega^{*}=1$ and $\eta_{0} / \eta_{i}=1$.

the increasing distance between two runners on a round racetrack.

Figs. $3 \mathrm{a}$ and $3 \mathrm{~b}$ reveal that $\mu_{m}^{*}$ and $\Theta$ decrease with the increase in $H$. This is because if the volume fraction of droplets is large, the space between droplets becomes narrow. In this case, since the electric double layer is confined to a narrow space, the time necessary to adjust the ionic distribution inside is short, and the phase lag is small. The influence of the thickness of the double layer surrounding a droplet on its electrophoretic behavior is also illustrated in Fig. 3. In the limit, as $\kappa a \rightarrow 0$, the magnitude of the dynamic mobility always decrease to zero. This is because as $\kappa a \rightarrow 0$, indicating an infinitely thick double layer, it influences all the other droplets throughout the entire 


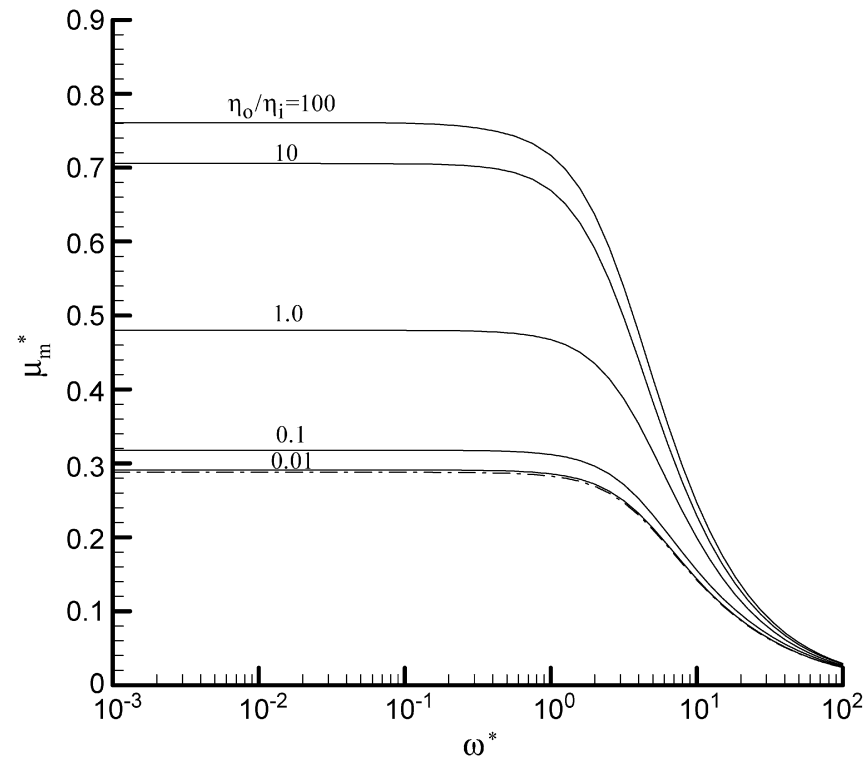

(a)

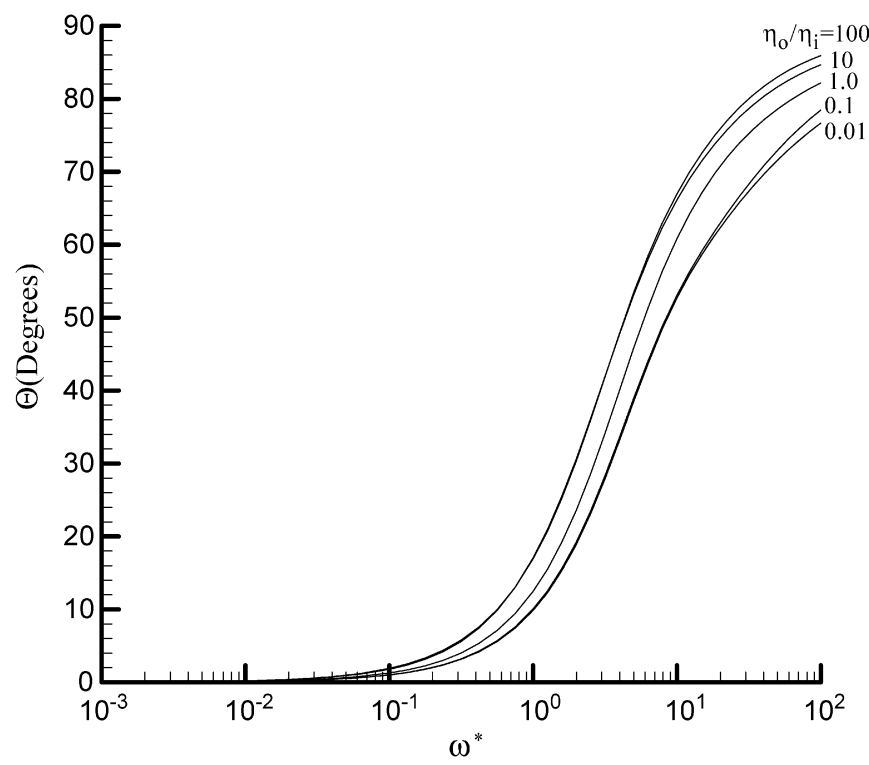

(b)

Fig. 4. Variation of scaled magnitude of electrophoretic mobility $\mu_{m}^{*}$ (a), and phase angle $\Theta(\mathrm{b})$, as a function of $\omega^{*}$ for various values of $\left(\eta_{0} / \eta_{i}\right)$ at $\kappa a=1$ and $H=0.1$.

electrolyte dispersion system. Due to the double-layer overlap effect, it actually yields a uniform distribution of ions and thus electric potential throughout. As a result, no electric force is generated upon the droplet and it remains stationary. However, the effect of the double-layer overlap gradually disappears with the increase of $\kappa a$; that is, the decrease of double-layer thickness yield a nonuniform distribution of ions, which sets up the motion eventually. The higher the $\kappa a$, the less significant the double-layer overlap from neighboring droplets, and the mobility increases accordingly. The phase lag, $\Theta$, decreases with increasing $\kappa a$, because now it is easier to react to the applied alternating electric field, with less entanglement from the double-layer overlap. This phenomenon gets less significant as $H$ is increased, because of the associated retarding hindrance

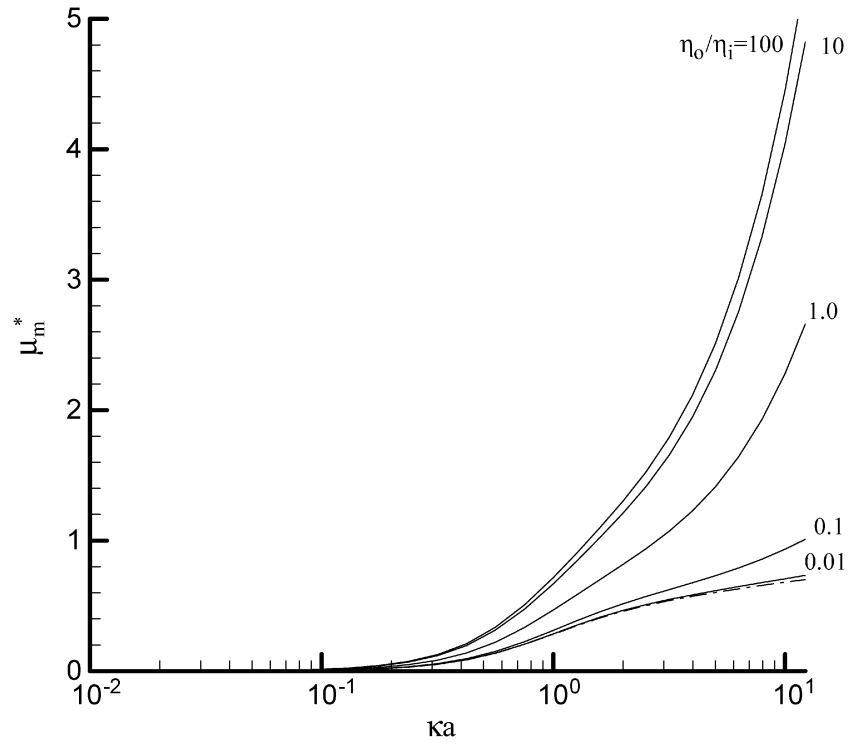

(a)

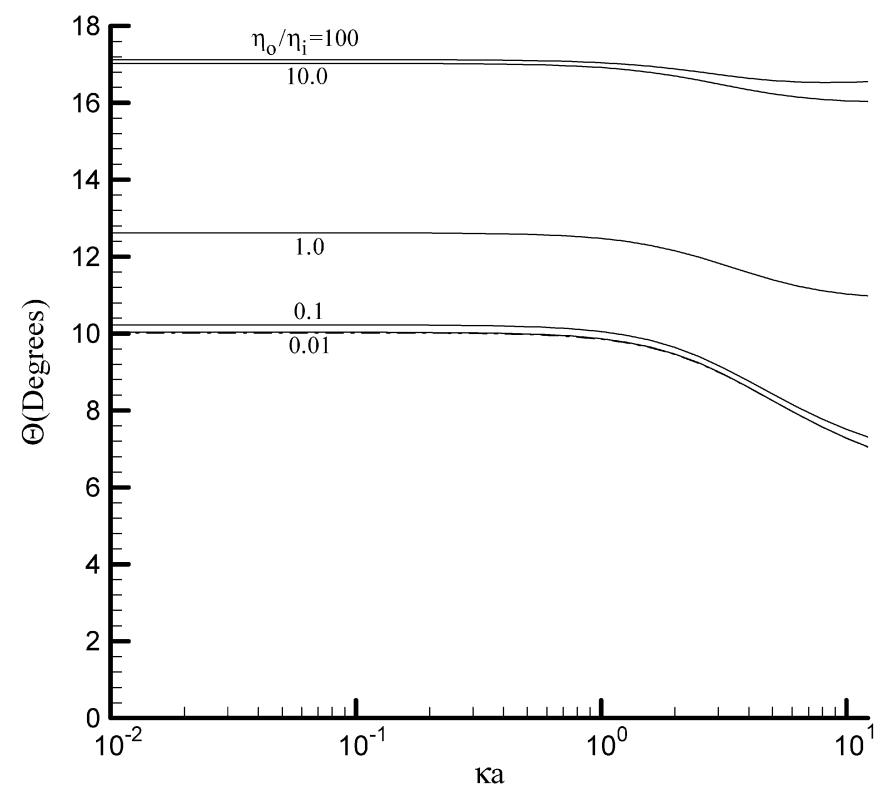

(b)

Fig. 5. Variation of scaled magnitude of electrophoretic mobility $\mu_{m}^{*}$ (a), and phase angle $\Theta(\mathrm{b})$, as a function of $\kappa a$ for various values of $\left(\eta_{0} / \eta_{i}\right)$ at $\omega^{*}=1$ and $H=0.1$.

effect, which appears to be more dominant in concentrated dispersions.

The influence of the viscosity of the droplet fluid on its electrophoretic behavior is illustrated in Figs. 4 and 5. These figures reveal that the larger the ratio $\left(\eta_{0} / \eta_{i}\right)$, that is, the more viscous the bulk fluid is relative to the droplet fluid, the larger the $\mu_{m}^{*}$. This is because the smaller the viscosity of the droplet fluid, the smaller the drag acting on a droplet. For comparison, the corresponding result for hard spherical particles [13] is also presented in Figs. 4 and 5. As can be seen from the two figures, they serve as limiting cases. If $\left(\eta_{0} / \eta_{i}\right)$ is equal to 0.01 , we can see that the dynamic electrophoretic mobility of liquid drops is very close to that of hard spheres. The phase lag is found to increase with the decrease in the viscosity of the droplet fluid 


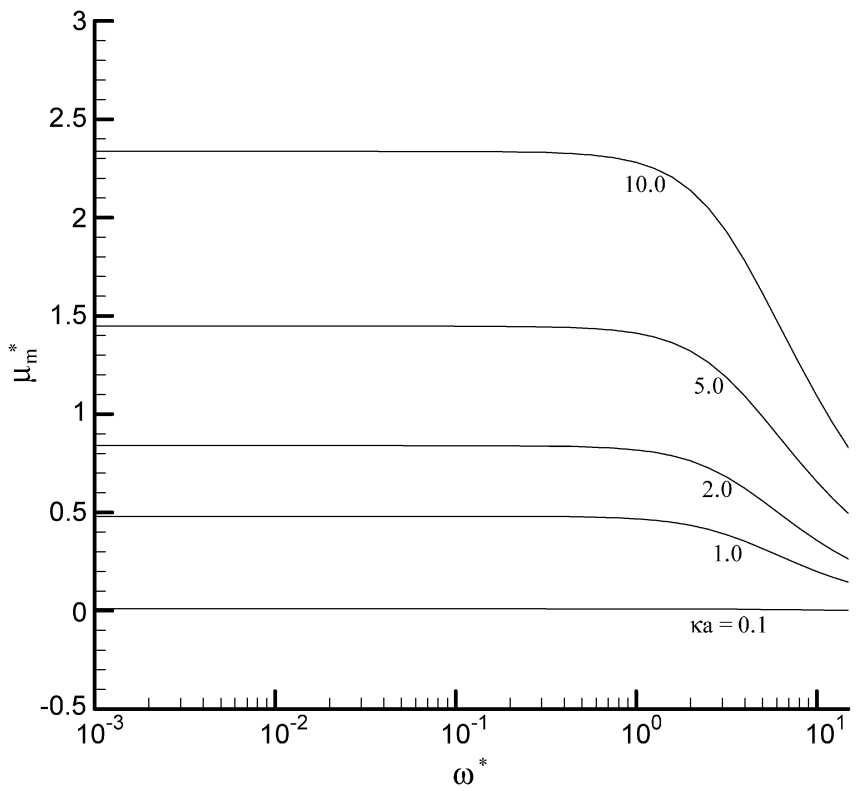

(a)

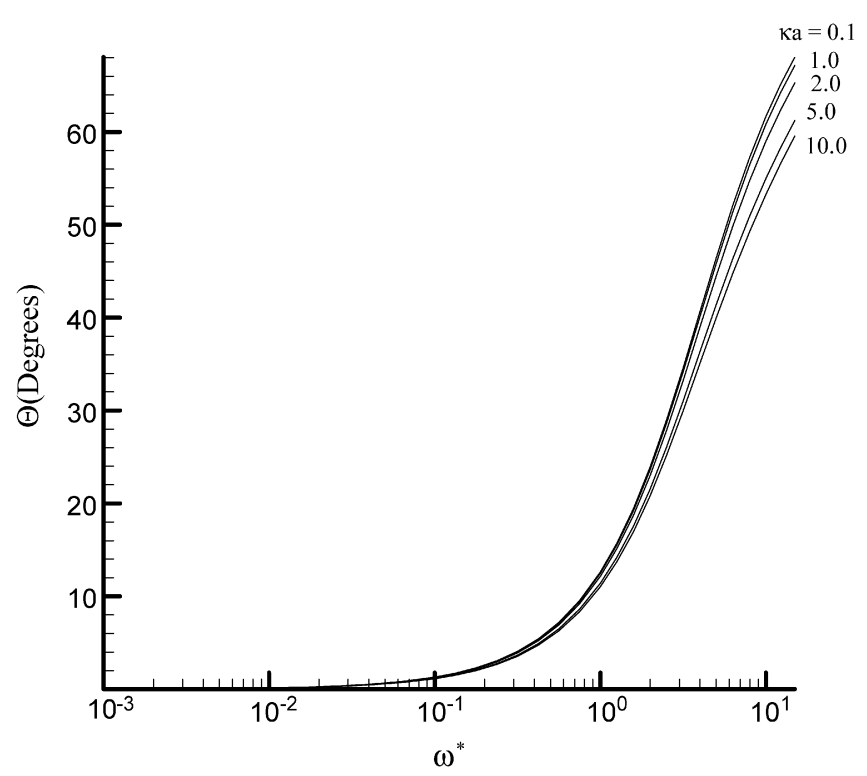

(b)

Fig. 6. Variation of scaled magnitude of electrophoretic mobility $\mu_{m}^{*}$ (a), and phase angle $\Theta(\mathrm{b})$, as a function of $\omega^{*}$ for various values of $\kappa a$ at $H=0.1$ and $\eta_{0} / \eta_{i}=1$.

(larger $\left.\left(\eta_{0} / \eta_{i}\right)\right)$. This is because a droplet with a smaller viscosity has a larger $\mu_{m}$ and inertia, and a longer time is needed for it to alter its direction of movement. The variations of $\mu_{m}$ and $\Theta$ with $\kappa a$ at various viscosity ratios in Fig. 5 are mainly due to the double-layer overlap effect, as we deduced before in Fig. 3.

The influence of the thickness of the double layer surrounding a droplet on its electrophoretic behavior is also illustrated in Fig. 6. This figure indicates that $\mu_{m}^{*}$ increases with the increase in $\kappa a$ for a fixed frequency. As we said before, the overlap of neighboring double layers has the effect of restraining the movement of droplets. When $\kappa a=0.1$, for example, the overlap effect is so severe that $\mu_{m}^{*}$ approaches zero, which results in a nearly horizontal line along varying $\omega^{*}$ in Fig. 6a. Similarly, the phase lag declines with the increase in $\kappa a$ because the thinner the double layer, the more easily it moves accordingly.

In summary, the dynamic electrophoretic behavior of a droplet dispersion is analyzed under conditions of low surface potential and an arbitrary volume fraction of droplets and double layer thickness. We conclude the following: (i) If the effect of double-layer polarization is neglected, as in the current case of low zeta potential, the phase of the electrophoretic velocity of a droplet, and consequently its mobility, is behind that of the applied electric field. (ii) The magnitude of electrophoretic mobility declines with the volume fraction of droplets. (iii) The smaller the viscosity of the droplet fluid the larger the magnitudes of the electrophoretic mobility and the phase lag. As the viscosity of the droplet fluid increases, the electrophoretic behavior of a droplet approaches that of a rigid particle. (iv) The magnitude of electrophoretic mobility increases, but the corresponding phase lag decreases, with the decline in the thickness of the double layer.

\section{Acknowledgment}

This work is supported by the National Science Council of the Republic of China.

\section{References}

[1] R.J. Hunter, Colloids Surf. A 141 (1998) 37.

[2] R.W. O’Brien, J. Fluid Mech. 190 (1988) 71.

[3] R.P. Sawatzky, A.J. Babchin, J. Fluid Mech. 246 (1993) 321.

[4] C.S. Mangelsdorf, L.R. White, J. Chem. Soc. Faraday Trans. 88 (1992) 3567.

[5] H. Ohshima, J. Colloid Interface Sci. 179 (1996) 431.

[6] H. Ohshima, J. Colloid Interface Sci. 195 (1997) 137.

[7] H. Ohshima, Colloids Surf. A 149 (1999) 5.

[8] H. Ohshima, Colloids Surf. A 159 (1999) 293.

[9] S. Levine, G.H. Neale, J. Colloid Interface Sci. 47 (1974) 520.

[10] A.S. Dukhin, V. Shilov, Y. Borkovskaya, Langmuir 15 (1999) 3452.

[11] V.N. Shilov, N.I. Zharkikh, Yu.B. Borkovskaya, Colloid J. 43 (1981) 434.

[12] E. Lee, F.Y. Yen, J.P. Hsu, J. Phys. Chem. B 105 (2001) 7239.

[13] J.P. Hsu, E. Lee, F.Y. Yen, J. Phys. Chem. B 106 (2002) 4789.

[14] E. Lee, C.H. Fu, J.P. Hsu, J. Colloid Interface Sci. 250 (2002) 327.

[15] C.P. Tung, E. Lee, J.P. Hsu, J. Colloid Interface Sci. 260 (2003) 118.

[16] S.H. Lou, E. Lee, J.P. Hsu, J. Colloid Interface Sci. 285 (2005) 865.

[17] W.L. Min, E. Lee, J.P. Hsu, J. Colloid Interface Sci. 299 (2006) 464.

[18] F. Booth, J. Chem. Phys. 19 (1951) 1331.

[19] V.G. Levich, Physicochemical Hydrodynamics, Prentice Hall, Englewood Cliffs, NJ, 1962.

[20] S. Levine, R.N. O’Brien, J. Colloid Interface Sci. 43 (1973) 616.

[21] S. Levine, in: G.E. Rindone (Ed.), Materials Proceeding in the Reduced Gravity Environment of Space, vol. 9, North-Holland, 1981, p. 241.

[22] E. Lee, W.L. Min, J.P. Hsu, Langmuir 22 (2006) 3920.

[23] S. Kuwabara, J. Phys. Soc. Jpn. 14 (1959) 527.

[24] E. Lee, J.W. Chu, J.P. Hsu, J. Colloid Interface Sci. 205 (1998) 65.

[25] E. Lee, J.W. Chu, J.P. Hsu, J. Colloid Interface Sci. 209 (1998) 240.

[26] J. Happel, H. Brenner, Low-Reynolds Number Hydrodynamics, Nijhoff, Boston, 1983. 